\title{
Heparan sulphate synthetic and editing enzymes in ovarian cancer
}

\author{
AC Backen*,', CL Cole', SC Lau', AR Clamp', R McVey², JT Gallagher' and GC Jayson' \\ 'Department of Medical Oncology, Paterson Institute for Cancer Research, Christie Hospital, Cancer Research UK and University of Manchester, \\ Wilmslow Road, Manchester M20 4BX, UK; '² Department of Histopathology, St Mary's Hospital, Oxford Road, Manchester MI 3 OJH, UK
}

Several angiogenic growth factors including fibroblast growth factors I and 2 (FGFI and FGF2) depend on heparan sulphate (HS) for biological activity. We previously showed that all cellular elements in ovarian tumour tissue synthesised HS but biologically active HS (i.e. HS capable of binding FGF2 and its receptor) was confined to ovarian tumour endothelium. In this study, we have sought to explain this observation. Heparan sulphate sulphotransferases I and 2 (HS6STI and HS6ST2) attach sulphate groups to C-6 of glucosamine residues in HS that are critical for FGF2 activation. These enzymes were strongly expressed by tumour cells, but only HS6STI was found in endothelial cells. Immunostaining with the 3GI0 antibody of tissue sections pretreated with heparinases indicated that HS proteoglycans were produced by tumour and endothelial cells. These results indicated that, in contrast to the endothelium, HS produced by tumour cells may be modified by cell-surface heparanase (HPAI) or endosulphatase (SULF). Protein and RNA analysis revealed that HPA I was strongly expressed by ovarian tumour cells in eight of ten specimens examined. HSULF-I, which removes specific 6-O-sulphate groups from HS, was abundant in tumour cells but weakly expressed in the endothelium. If this enzyme was responsible for the lack of biologically active HS on the tumour cell surface, we would expect exogenous FGF2 binding to be preserved; we showed previously that this was indeed the case although FGF2 binding was reduced compared to the endothelium and stroma. Thus, the combined effects of heparanase and HSULF could account for the lack of biologically active HS in tumour cells rather than deficiencies in the biosynthetic enzymes.

British Journal of Cancer (2007) 96, I544- I548. doi:I0.I038/sj.bjc.6603747 www.bjcancer.com

Published online 17 April 2007

(c) 2007 Cancer Research UK

Keywords: ovarian cancer; HS; FGF; heparanase; HSULF-I

The extracellular matrix (ECM) is composed of a network of macromolecules that maintain tissue and cellular architecture. Heparan sulphate (HS) proteoglycans (HSPGs) are major components of the ECM and of cell-surface membranes, and they play critical roles in regulating the flow of information between cells and their microenvironment (Blackhall et al, 2001). In addition to anchoring cells to matrix fibres and maintaining tissue structure, HSPGs serve at least two additional biological activities. First, the HS chains of these PGs bind to many growth factors and cytokines such as fibroblast growth factors (FGFs), vascular endothelial growth factor and transforming growth factor (Lyon et al, 1997; Robinson and Stringer, 2001; Ostrovsky et al, 2002, respectively). This binding confers on the growth factors resistance to proteolysis and biological stability and provides a reservoir of growth and migration factors that can be mobilised in accordance with physiological demand. Second, cell-surface HSPGs act as mandatory co-receptors, facilitating binding of growth factors to their cognate high-affinity signal-transducing receptors (reviewed in Gallagher, 2001; Presta et al, 2005).

Fibroblast growth factor 2 is a potent angiogenic cytokine, which is dependent on HS for its biological activity (Yayon et al, 1991,

*Correspondence: Dr AC Backen;

E-mail: Alison.Backen@Manchester.ac.uk

Revised 27 February 2007; accepted 20 March 2007; published online 17 April 2007
Chang et al, 2000). In a previous study of HSPGs, we showed that stromal syndecan 1 was an adverse prognostic factor for ovarian cancer and that syndecan 3 was unusually expressed by the vasculature (Davies et al, 2004). However, the majority of the function of the HSPGs is mediated by the structure of the HS glycosaminoglycan component (Jayson et al, 1998). Therefore, in a second study, we investigated the relationship between HS, FGF-2 and the signal-transducing receptors in human, advanced-stage, serous ovarian adenocarcinoma. Using a unique molecular probe, FR1c-AP, which consisted of a soluble FGF receptor 1 isoform IIIc covalently linked to an alkaline phosphatase moiety, the distribution of HS that had the ability to support the formation of an HS/ FGF-2/FR1c-AP complex was determined (Ornitz et al, 1992; Chang et al, 2000). This may be taken as a surrogate marker for the distribution of biologically active HS. In ovarian cancer tissue, we found that this probe bound predominantly to endothelial cells and stroma but not adenocarcinoma cells (Whitworth et al, 2005). In the current study, we therefore tested the hypothesis that the enzymes that sulphate HS, such as the sulphotransferases and the HS-cell-surface editing enzymes, such as the endosulphatases and endoheparanase (HPA1), determine the distribution of FGF2activating HS.

Heparan sulphate chains are synthesised as a post-translational modification of specific core proteins in the Golgi by enzymes that initially polymerise a chain of repeating glucuronic acid (GlcA) and GlcNAc disaccharides, which are then variously modified by $\mathrm{N}$ - and $\mathrm{O}$-sulphation and hexuronic acid epimerisation, converting glucuronic acid (GlcA) into iduronic acid (Lindahl et al, 1998). 
These modifications occur in clusters, creating polymorphic sulphated domains (S-domains) that form the principal recognition sites for heparin/HS-binding proteins.

A key modification of HS is carried out by the small family of 6-O-sulphotransferases (HS6ST) that catalyse the transfer of sulphate from adenosine 3'-phosphate, 5'-phosphosulphate to C-6 (exocyclic carbon) of the glucosamine residue in HS, thereby providing functional groups that are critical to the formation of the trimolecular signalling complex: FGF2, FGFR and HS (Pye et al, 1998; Robinson et al, 2005; Ai et al, 2006). The S-domains containing 6-O-sulphate groups can be excised from the HS chain by cell-surface heparanase (HPA1), a $\beta$-endoglycosidase that releases saccharide fragments from HSPG core proteins. Alternatively, the fine structure of HS can be modified by endosulphatases that specifically remove 6-O-sulphate groups from HS-S-domains (Morimoto-Tomita et al, 2002; Viviano et al, 2004). This has functional consequences as, in quails, QSULF-1, the avian homologue of mammalian SULFs, has dual regulatory functions as a negative regulator of FGF signalling and a positive regulator of Wnt signalling (Ai et al, 2003; Wang et al, 2004)

Heparanase mRNA expression has been investigated in a wide variety of human tumours (reviewed in Simizu et al, 2004). Kodama et al (2003) examined heparanase RNA expression by RT-PCR in five borderline and 31 malignant epithelial ovarian tumours. Heparanase mRNA expression was present in 16 of 31 malignant epithelial ovarian tumours. In contrast, in the five borderline epithelial ovarian tumours, heparanase mRNA was not detected.

In the current study, we have investigated why biologically active HS is largely restricted to the ovarian tumour endothelium. The data suggest that tumour cells synthesise HS, but its binding properties may be modified by SULF action. Moreover, the pattern of expression of heparanase reveals the potential for localised degradation of tumour-associated HS, and release of active HS saccharide-growth factor complexes that could enhance the malignant phenotype.

\section{MATERIALS AND METHODS}

\section{Tissue samples}

Tumours from 10 patients with serous ovarian carcinomas were investigated; these were a mixture of well, moderately and poorly differentiated tumours. Two normal ovaries were also examined. These were taken from consenting patients, under the ethical permission of South Manchester Research Ethics Committee.

\section{Riboprobe preparation}

Specific riboprobes for HS6ST1, HS6ST2, HPA1 and HSULF-1 were prepared by amplifying gene-specific fragments, identified using the NCBI Blast programmes, from normal ovarian RNA using the primers as follows:

HS6ST1 forward 5'-AAAGATATCATGGTTGAGCGCCGC- ${ }^{\prime}$ and reverse $5^{\prime}$-CTCGCGGACCGGGAAGTAG-3', HS6ST2 forward $5^{\prime}$ GAATTCGGCCAGGCGAAAGCGTC- $3^{\prime}$ and reverse $5^{\prime}$-GTCGACCG CCATTTCTCTACACTG-3', HPA1 forward 5'-TTCGATCCCAA GAAGGAATCAAC- $3^{\prime}$ and reverse $5^{\prime}$-GTAGTGATGCCATGTAACT GAATC-3', HSULF-1 forward 5'-GGATCCCCTTCCACGCTCTGG CCGATTG- $3^{\prime}$ and reverse $5^{\prime}$-GGATCCATCCAACAGTCAAAT CACTTGCCCAAAT- $3^{\prime}$.

These were cloned into the pSPT19 vector (Roche, Mannheim, Germany) and the sequence was verified. The vector was linearised, rendered RNAase-free by phenol/chloroform/isoamyl alcohol purification and used as a template for in vitro transcription of digoxigenin-labelled antisense or sense (control) riboprobes using a SP6/T7 transcription kit (Roche).

\section{ISH method}

Tissue sections were dewaxed and rehydrated, denaturated with $0.2 \mathrm{M} \mathrm{HCl}$ for $20 \mathrm{~min}$ and then digested with proteinase $\mathrm{K}$ $\left(5 \mu \mathrm{g} \mathrm{ml}^{-1}\right)$ at $37^{\circ} \mathrm{C}$ for $30 \mathrm{~min}$. Slides were hybridised with the anti-sense probe or sense probe (negative control) at concentrations of $1-2 \mu \mathrm{g} \mathrm{ml}^{-1}$ in hybridisation buffer (Sigma-Aldrich, Gillingham, UK). After hybridisation, washes and incubation with anti-digoxigenin antibody and visualisation were carried out using NBT/BCIP (Roche) according to the manufacturer's instructions. Sections were fixed, mounted and examined with an Olympus BX51 light microscope (Olympus, Southall, UK) using a $\times 40$ plan neofluar, 1.35 NA, oil immersion objective lens. Visualisation was carried out utilising a Progress C14 camera (Jenoptik, Jena, Germany) via Adobe PhotoShop 7.

\section{Streptavidin ABC/HRP Immunohistochemistry}

Tissue sections were dewaxed and rehydrated. Antigen retrieval was performed in citrate buffer (pH6), using a microwave oven. For 3G10 staining only, sections were pre-treated with heparinases I and II (Grampian Enzymes, Orkney, UK) at a total of $5 \mathrm{mU} \mathrm{ml}^{-1}$ in $50 \mathrm{~mm}$ sodium acetate, $0.5 \mathrm{mM}$ calcium acetate $(\mathrm{pH} 7.0)$, for $4 \mathrm{~h}$ at $37^{\circ} \mathrm{C}$, replenishing after $2 \mathrm{~h}$. To demonstrate the specificity of 3 G10 binding, a negative control section was included in which the heparinases I and II digestion step had been omitted. Endogenous peroxidase activity was blocked by incubation in $0.8 \% \mathrm{H}_{2} \mathrm{O}_{2}$ in methanol for $10 \mathrm{~min}$. A blocking solution of $10 \%$ goat serum in TBS was applied for $10 \mathrm{~min}$. Sections were incubated overnight at $4{ }^{\circ} \mathrm{C}$ with either anti-HS monoclonal antibody 10E4 (Seikagaku Corporation, Tokyo, Japan) at a dilution of $1: 200\left(5 \mu \mathrm{g} \mathrm{ml}^{-1}\right.$ final concentration), anti $\Delta$-HS monoclonal antibody $3 \mathrm{G} 10$ (Seikagaku Corporation) at a dilution of $1: 250\left(4 \mu \mathrm{g} \mathrm{ml}^{-1}\right.$ final concentration), or anti-HPA1 monoclonal antibody (Insight Biopharmaceuticals, Rehovot, Israel) at a dilution of $1: 60\left(670 \mu \mathrm{g} \mathrm{ml}^{-1}\right.$ final concentration). An equivalent concentration of non-immune mouse serum was used as a negative control (DAKO, Kyoto, Japan). Sections were incubated with biotinylated rabbit antimouse antibody $\left(1: 400 ; 2 \mathrm{ng} \mathrm{ml}^{-1}\right.$ final concentration; DAKO), and with peroxidase-labelled streptavidin (DAKO). Colour was developed using 3,3'-diaminobenzidine (Sigma-Aldrich, USA). Counterstaining was performed with Gill's haematoxylin. Samples were evaluated by microscopy and semiquantitatively analysed for heparanase expression (intense, moderate or absent staining).

\section{RESULTS}

We have used two in situ techniques to investigate the restricted distribution of biologically active HS to the tumour endothelium; the first question we addressed was whether there was evidence that HS synthesis occurred in tumours. We know that the molecular probe used to detect biologically active HS has an absolute requirement for the presence of 6-O-sulphate groups (Pye et al, 1998) and, therefore, we examined the distribution of the enzymes that catalyse the attachment of that moiety, the HS-6-O-sulphotransferases.

\section{In situ hybridisation locates HS6ST1 and HS6ST2 mRNA}

Specific digoxigenin-labelled riboprobes for HS6ST1 and HS6ST2 mRNA were generated and used to investigate ten serous carcinomas and two normal ovaries. Our ability to study normal ovarian epithelium was compromised by the poor preservation of cellular architecture. Figure 1A shows HS6ST1 mRNA to be present in ovarian cancer and endothelial cells, but absent in stroma; all 10 tumours stained with similar intensities. Figure 1B shows HS6ST2 RNA to be present in ovarian cancer cells, but absent in endothelial cells and stroma and again all 10 tumours stained with similar 

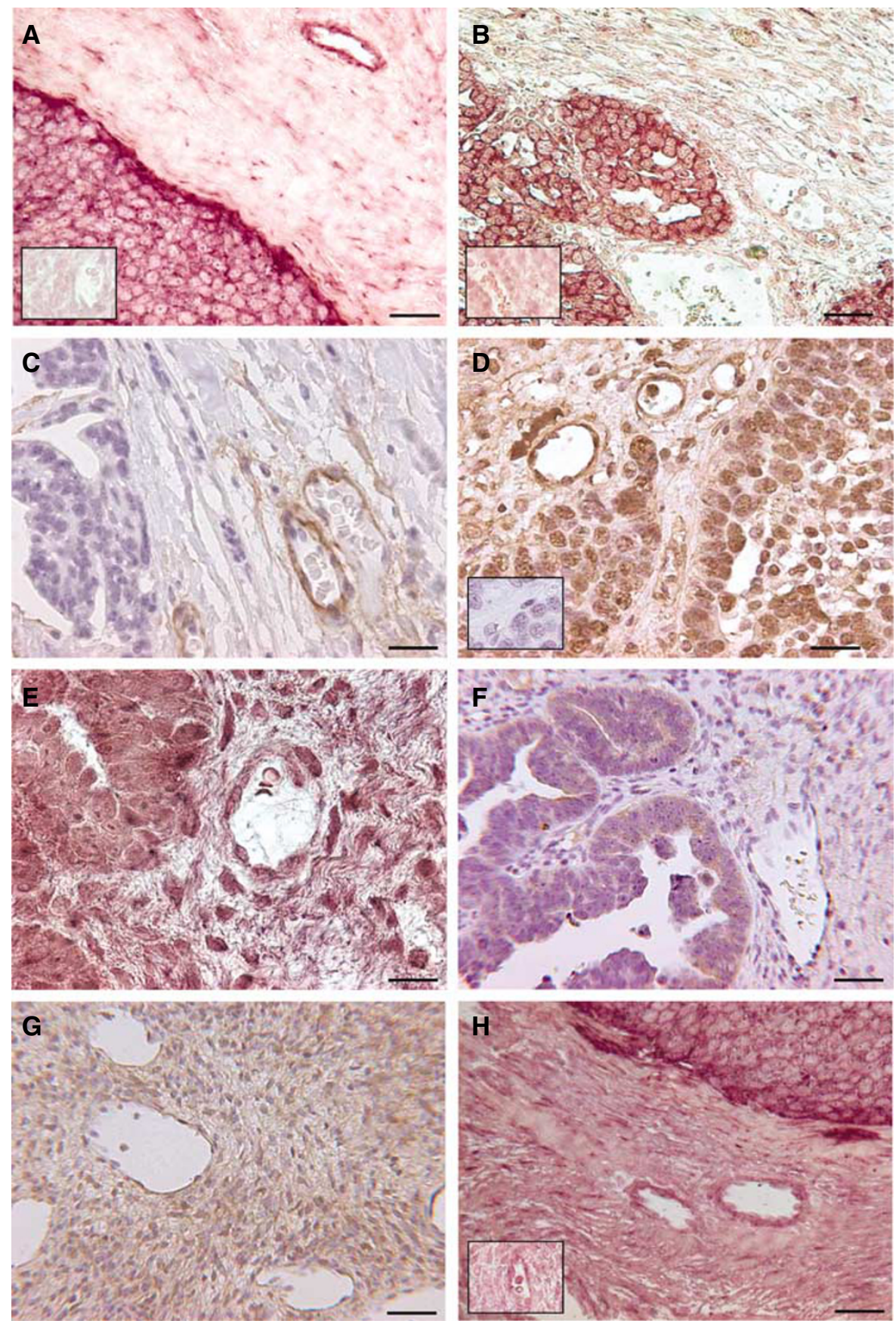

Figure I (A) HS6STI ISH shows RNA to be present in ovary tumour and endothelial cells, but absent in stroma and normal ovary (inset). (B) HS6ST2 ISH shows RNA to be present in ovary tumour cells, but absent in endothelial cells, stroma and normal ovary (inset). (C) IOE4, an anti-HS monoclonal antibody was used to demonstrate that intact HS chains were only present on endothelial cells in ovarian tumours. (D) $3 \mathrm{G} I 0$ antibody was used to detect the HS stubs that remain after heparinase digestion. A negative control shows $3 \mathrm{Gl} 0$ staining to be negative without prior heparinase digestion (inset). (E) Heparanase ISH shows RNA to be present in ovary tumour cells, but absent in endothelial cells and stroma. (F) Heparanase IHC shows protein to be present in ovary tumour cells, but absent in endothelial cells and stroma. (G) Heparanase IHC on normal human ovary shows protein to be present at a low level in most cells. (H) HSULF-I ISH shows RNA to be present in ovary tumour cells, but absent in endothelial cells, stroma and normal ovary (inset). Each scale bar represents $400 \mu \mathrm{m}$.

intensities. Both HS6ST1 and HS6ST2 mRNAs were expressed at higher levels in tumour than in the normal ovaries examined (Figure $1 \mathrm{~A}$ and $\mathrm{B}$, inset).

We extended these studies by examining the distribution of the mRNA for 2-O-sulphotransferase and this closely mirrored that seen with the HS6ST2 mRNA, again suggesting that sulphated HS was made by ovarian cancer cells (data not shown).

\section{HS staining}

In our previous study (Whitworth et al, 2005), which showed that biologically active HS was expressed by the endothelium (inferred by the binding of the FR1c-AP probe), the implication was that HS6ST1 would be confined to the endothelium. However, the above results contradict that hypothesis. Therefore, we carried out 
a series of IHC studies to determine whether HSPGs were expressed by the ovarian cancer cells.

The same 10 serous carcinomas and 2 normal ovaries were examined for the presence of HS using IHC. Figure 1C shows 10E4 staining, which indicated that intact sulphated HS chains were only present on endothelial cells in ovarian tumours. However Figure 1D shows $3 \mathrm{G} 10$ antibody staining, which detects the HS stubs that remain on the PG core protein after heparinase depolymerisation. The inset picture shows that $3 \mathrm{G} 10$ does not stain when the heparinase digestion step is omitted. With both 10E4 and 3G10, all 10 tumours stained with similar intensity. These experiments showed that carcinoma cells strongly bind to $3 \mathrm{G} 10$, suggesting that HSPGs were made by tumour cells, whereas the HS was subsequently degraded or modified to eliminate the 10E4 epitope.

\section{Investigation of heparanase (HPA1) mRNA and protein expression}

We have shown that the HS6ST1 and 2 and HS2-OST biosynthetic enzyme RNA, essential for biologically active HS, were expressed by ovarian cancer cells although the HS 10E4 epitope, often used as a marker of HS, was not present on the cell surface. To investigate this further, we hypothesised that heparanase was present and may be responsible for the lack of $10 \mathrm{E} 4$ reactivity. A specific digoxigeninlabelled riboprobe for HPA1 was generated and used to investigate our panel of 10 serous carcinomas and two normal ovaries. Figure 1E shows heparanase RNA to be present in ovarian cancer cells, but absent in endothelial cells and stroma. Two of the 10 tumour specimens showed a lower expression of heparanase RNA. Because of the extensive processing of heparanase, we also used IHC and this localised HPA1 protein (Figure 1F) to tumour cells, but did not detect the enzyme in endothelial cells and only at a low level in stroma. All 10 tumours examined showed similar patterns of protein staining. In normal human ovary, immunohistochemistry showed that heparanase protein was present at a low level in most cells (Figure 1G). These experiments demonstrate that heparanase was strongly expressed by ovarian cancer cells, but was virtually absent from endothelial cells and normal ovarian tissues.

\section{In situ hybridisation locates HSULF-1 mRNA}

HSULF-1 is an enzyme that removes 6-O-sulphate residues from cell-surface HS. Although the heparanase data might account for the distribution of biologically active HS, another possibility was that HSULF-1 might be responsible.

A specific digoxigenin-labelled riboprobe for HSULF-1 was generated and used to investigate ten serous carcinomas and two normal ovaries. Figure $1 \mathrm{H}$ shows HSULF-1 mRNA to be strongly expressed in ovarian tumour cells, but expressed at a much lower level in endothelial cells and stroma. The staining intensity was similar in all 10 tumours examined. HSULF-1 was expressed at higher levels in tumour than in the normal ovaries examined (Figure $1 \mathrm{H}$, inset).

\section{DISCUSSION}

In previous studies, we showed that stromal syndecan 1 was an adverse prognostic factor in ovarian cancer and that syndecan 3, which is usually found on neuronal tissue, was aberrantly expressed in the tumour vasculature (Davies et al, 2004). In a further investigation, we used a novel molecular probe, which demonstrated that endothelial HS was able to support receptor engagement when FGF2 was present; in other words, HS had the capacity to activate FGF2 (Whitworth et al, 2005). In this study we sought to explain the mechanism.

The findings from this study are summarised in Table 1. The HS epitope recognised by the antibody $10 \mathrm{E} 4$ was only detected on
Table I Summary of results

\begin{tabular}{|c|c|c|c|}
\hline & Tumour & Stroma & Endothelium \\
\hline Endogenous FGF2 ${ }^{\mathrm{a}}$ & Low & Low & Moderate \\
\hline FGF2 binding $^{a}$ & Mild & Moderate & Moderate-strong \\
\hline FRIC-APa & None & Weak & Moderate-strong \\
\hline HPAI & +++ & + & - \\
\hline HSULF-I & +++ & + & + \\
\hline HS6STI & +++ & + & +++ \\
\hline HS6ST2 & +++ & + & - \\
\hline Intact HS (IOE4) & - & - & +++ \\
\hline HS stubs (3GIO) & +++ & + & +++ \\
\hline
\end{tabular}

aFindings of Whitworth et al (2005)

endothelial cells within ovarian tumour sections. Although the mRNAs of the synthetic enzymes were present in cancer cells, it was unclear whether HSPGs were expressed on the cell surface. By treating the tissues with heparinases to degrade cell-surface HS and then examining the distribution of bound, exogenously added 3 G10 antibody, we were able to test whether the HSPG core proteins were present on the tumour-cell surface. These data showed widespread binding of the antibody in the tissue sections, suggesting that in cancer cells as well as in other cell populations present within the tumour HS-bearing PGs were expressed on the plasma membrane.

In a previous study (Whitworth et al, 2005), we showed that in ovarian tumours the distribution of biologically active HS revealed by the colocalisation of FGF2 and the FGF-receptor probe (FR1cAP) was confined to the tumour endothelium. Whereas FGF2 binds to $\mathrm{N}$ and 2-O sulphated S-domains in HS, biologically active S-domains contain 6-O-sulphate moieties (Kan et al, 1993; Lindahl et al, 1998; Pye et al, 1998). Thus, the difference in binding requirements for the FR1c-AP probe and exogenous FGF2 is 6-Osulphate on glucosamine; by comparing the distribution of the two probes we can examine the distribution of 6-O-sulphate. This is the moiety removed by the HSULF enzymes that we show here to be expressed strongly by tumour cells but weakly by the endothelial cells. Although our data apparently contradict those of Lai et al (2003), the differences have most likely arisen because in our study we determined the patterns of cellular expression of HSULF on tissue sections rather than PCR analysis of total tumour mRNA. However, our data also demonstrate that tumour cells and endothelial cells can be distinguished by their expression of HPA1; the high levels of this enzyme on tumour cells compared to its weak expression on the endothelium is likely to contribute to the differential reactivities of the HS found on the surfaces of these two cell populations. Thus, the combined effects of HSULF and HPA1 are probably responsible for the restricted distribution of biologically active cell-surface HS in ovarian cancer. These findings indicate that in ovarian tumours, FGF2 does not act directly on the malignant cells, rather it acts indirectly by promoting tumour angiogenesis.

Heparanase has been strongly correlated with metastatic potential in human cancer probably by disrupting the structure of basement membranes and by releasing HS saccharide growth factor complexes from the pericellular matrix enabling their unrestricted access to cell-surface receptors (Elkin et al, 2001). Our data again reinforce the importance of HS to the malignant phenotype and highlight the potential therapeutic advantage of targeting heparanase in human cancer.

\section{ACKNOWLEDGEMENTS}

We thank Garry Ashton and Caron Abbey for their histological assistance, and Steve Bagley for his help with microscopy and photography. 


\section{REFERENCES}

Ai X, Do AT, Lozynska O, Kusche-Gullberg M, Lindahl U, Emerson Jr CP (2003) QSulf1 remodels the 6-O sulfation states of cell surface heparan sulfate proteoglycans to promote Wnt signaling. J Cell Biol 162(2): 341 - 351, doi:10.1083/jcb.200212083

Ai X, Do AT, Kusche-Gullberg M, Lindahl U, Lu K, Emerson Jr CP (2006) Substrate specificity and domain functions of extracellular heparan sulfate 6-O-endosulfatases, QSulf1 and QSulf2. J Biol Chem 281(8): 4969-4976, doi:10.1074/jbc.M511902200

Blackhall FH, Merry CL, Davies EJ, Jayson GC (2001) HS proteoglycans and cancer. Br J Cancer 85: 1094 - 1098, doi:10.1054/bjoc.2001.2054

Chang Z, Meyer K, Rapraeger AC, Friedl A. (2000) Differential ability of heparan sulfate proteoglycans to assemble the fibroblast growth factor receptor complex in situ. FASEB J 14(1): 137-144

Davies EJ, Blackhall FH, Shanks JH, David G, McGown AT, Swindell R, Slade RJ, Martin-Hirsch P, Gallagher JT, Jayson GC (2004) Distribution and clinical significance of HS proteoglycans in ovarian cancer. Clin Cancer Res 10(15): 5178-5186

Elkin M, Ishai-Michaeli R, Friedmann Y, Papo O, Pecker I, Voldausky I (2001) Heparanase as mediator of angiogenesis: mode of action. FASEB J 15: 1661 - 1663, doi: 10.1096/fj.00-0895fje

Gallagher JT (2001) Heparan sulfate: growth control with a restricted sequence menu. J Clin Invest 108(3): 357-361

Jayson GC, Lyon M, Paraskeva C, Turnbull JE, Deakin JA, Gallagher JT (1998) HS undergoes specific structural changes during the progression from human colon adenoma to carcinoma in vitro. J Biol Chem 273(1): $51-57$

Kan M, Wang F, Xu J, Crabb JW, Hou J, McKeehan WL (1993) An essential heparin-binding domain in the fibroblast growth factor receptor kinase. Science 259: 1918 - 1921, doi: 10.1126/science.8456318

Kodama J, Shinyo Y, Hashen G, Hongo A, Yoshinouchi M, Hiramatsu Y (2003) Heparanase messenger RNA expression in epithelial ovarian tumor. Int J Mol Med 12(6): $961-964$

Lai J, Chien J, Staub J, Avula R, Greene EL, Matthews TA, Smith DI, Kaufmann SH, Roberts LR, Shridhar V. (2003) Loss of HSulf-1 upregulates heparin-binding growth factor signaling in cancer. J Biol Chem 278(25): 23107 -23117, doi:10.1074/jbc.M302203200

Lindahl U, Kusche-Gullberg M, Kjellén L (1998) Regulated diversity of HS. J Biol Chem 273: 24979-24982

Lyon M, Rushton G, Gallagher JT (1997) The interaction of the transforming growth factor-betas with heparin/HS is isoform-specific. J Biol Chem 272(29): $18000-18006$

Morimoto-Tomita M, Uchimura K, Werb Z, Hemmerich S, Rosen SD (2002) Cloning and characterization of two extracellular heparin- degrading endosulphatases in mice and humans. J Biol Chem 277(51): 49175 - 49185, doi:10.1074/jbc.M205131200

Ornitz DM, Yayon A, Flanagan JG, Svahn CM, Levi E, Leder P (1992) Heparin is required for cell-free binding of basic fibroblast growth factor to a soluble receptor and for mitogenesis in whole cells. Mol Cell Biol 12(1): $240-247$

Ostrovsky O, Berman B, Gallagher J, Mulloy B, Fernig DG, Delehedde M, Ron D (2002) Differential effects of heparin saccharides on the formation of specific fibroblast growth factor (FGF) and FGF receptor complexes. J Biol Chem 277: 2444-2453, doi:10.1074/jbc.M108540200

Presta M, Dell'Era P, Mitola S, Moroni E, Ronca R, Rusnati M. (2005) Fibroblast growth factor/fibroblast growth factor receptor system in angiogenesis. Cytokine Growth Factor Rev 16(2): 159-178, doi:10.1016/ j.cytogfr.2005.01.004

Pye DA, Vives RR, Turnbull JE, Hyde P, Gallagher JT (1998) HS oligosaccharides require 6-O-sulphation for promotion of basic fibroblast growth factor mitogenic activity. J Biol Chem 273(36): 22936-22942

Robinson CJ, Harmer NJ, Goodger SJ, Blundell TL, Gallagher JT. (2005) Cooperative dimerization of fibroblast growth factor 1 (FGF1) upon a single heparin saccharide may drive the formation of 2:2:1 FGF1.FGFR2c.heparin ternary complexes.. J Biol Chem 280(51): 42274-42282, doi:10.1074/jbc.M505720200

Robinson CJ, Stringer SE (2001) The splice variants of vascular endothelial growth factor (VEGF) and their receptors. J Cell Sci 114: 853-865

Simizu S, Ishida K, Osada H (2004) Heparanase as a molecular target of cancer chemotherapy. Cancer Sci 95(7): 553-558, doi:10.1111/j. 1349-7006.2004.tb02485.x

Viviano BL, Paine-Saunders S, Gasiunas N, Gallagher JT, Saunders S (2004) Domain-specific modification of heparan sulfate by QSULF1 modulates the binding of the bone morphogenetic protein antagonist noggin. J Biol Chem 279(7): 5604-5611, doi:10.1074/jbc.M310691200

Wang S, Ai X, Freeman SD, Pownall ME, Lu Q, Kessler DS, Emerson Jr CP (2004) QSulf1, a HS 6-O-endosulphatase, inhibits fibroblast growth factor signalling in mesoderm induction and angiogenesis. Proc Nat Acad Sci USA 101(14): 4833-4838, doi:10.1073/pnas.0401028101

Whitworth M, Backen A, Clamp A, Wilson G, McVey R, Friedl A, Rapraeger A, David G, McGown A, Slade R, Gallagher J, Jayson G (2005) Regulation of fibroblast growth factor-2 activity by human ovarian cancer tumor endothelium. Clin Cancer Res 11(12): 4282-4288

Yayon A, Klagsbrun M, Esko JD, Leder P, Ornitz DM (1991) Cell surface, heparin-like molecules are required for binding of basic fibroblast growth factor to its high affinity receptor. Cell 64(4): $841-848$, doi:10.1016/0092-8674(91)90512-W. 